\title{
Control Strategies for an Advanced Aircraft-Cabin Temperature-System
}

\author{
Alexander Pollok ${ }^{1,2}$
}

\begin{abstract}
Thermal regulation of aircraft cabins requires controlling the temperature of supplied fresh air. State of the art plant architectures support only a small number of temperature zones. In this paper we consider a novel architecture that allows an arbitrary number of temperature zones. This is bought at the expense of a more complex control problem. Control challenges connected to this novel architecture are identified and possible control approaches are presented. They are benchmarked against high-fidelity models in the equation-based object-oriented modelling language Modelica. Results show that control input normalization offers significant advantages for any kind of control system, while the choice between PIDbased and LQG-based control is somewhat ambiguous: The former shows better performance in the nominal case, the latter exhibits better robustness.
\end{abstract}

\section{INTRODUCTION}

In modern passenger aircraft the cabin temperature is usually regulated using a zone-based concept. Within a temperature-zone, the temperature of the air blown into the cabin is constant. A typical single-aisle aircraft features around two temperature zones for the passengers and one temperature zone for the flight deck. Cabin heat-loads are not homogeneous: Expensive travel classes like first class have a lower passenger-density compared to economy class, resulting in a lower area-specific heat-load. Galleys represent additional point-like heat-loads. These differences cannot be compensated by the control system, if they occur within the same temperature zone. This results in temperature deviations and degraded passenger comfort.

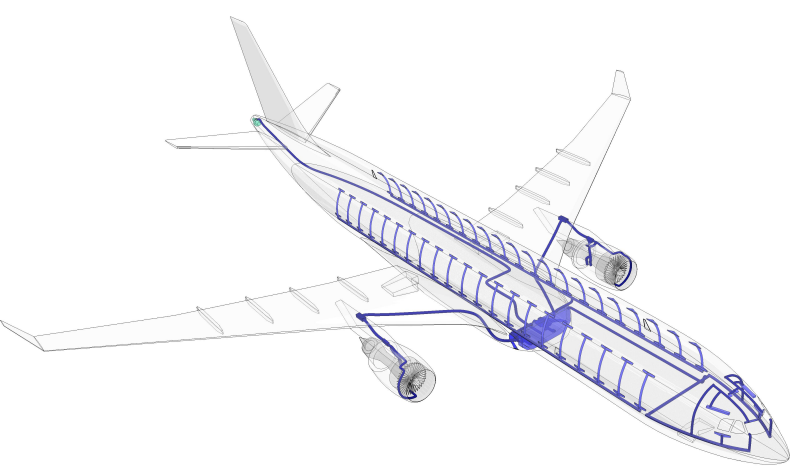

Fig. 1. Topography of an aircraft environmental control system

A conventional temperature regulation architecture is operating as follows: Hot bleed air from the compressor

\footnotetext{
${ }^{1}$ Institute of System Dynamics and Control, DLR German Aerospace Center, Wessling, Germany

${ }^{2}$ Dipartimento di Elettronica, Informazione e Bioingegneria, Politecnico di Milano, Milan, Italy

alexander.pollokedlr.de
}

stages of the engines is cooled to the target temperature in the air conditioning packs and ducted into the mixing chamber at a constant mass flow. For each cabin temperature zone a main duct is used to transport air from the mixing chamber to the respective temperature zone. For each main duct hot trim air is added to fine-tune air temperature to the requirement of the respective temperature zone. From the main duct riser ducts branch off in regular intervals and distribute the air into the cabin. Spent air leaves the cabin area through floor outlets into the underfloor area. From there, some of the air is filtered and recirculated into the mixing chamber. The remainder is vented into the environment through the outflow valve to regulate cabin pressure (see Figure 2).

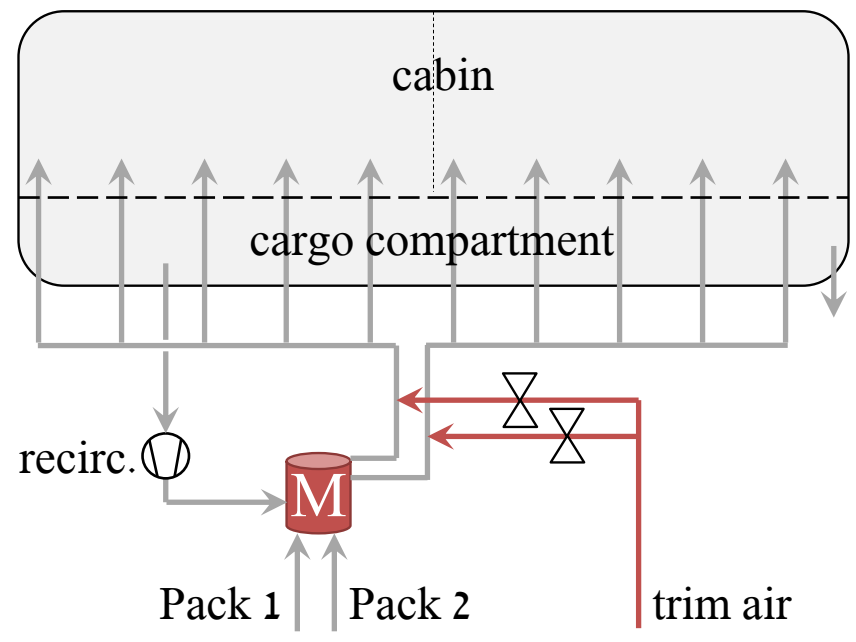

Fig. 2. Conventional cabin temperature regulation architecture (2 temperature zones)

Scalability of the conventional architecture is poor: Each temperature zone requires an additional main duct as well as corresponding thermal isolation. This results in an increase in weight and contruction space. Since contruction space is limited, the number of temperature zones is therefore also limited.

An alternative architecture is proposed in [1]: Here, an additional mixing chamber is utilized. Both chambers are supplied with a constant mass flow by different air conditioning packs, and kept at different temperatures. From both mixing chambers, one main duct is supplied that spans the complete length of the aircraft cabin. At the respective temperature zones, air from both ducts is locally mixed, see Figure 3. In this architecture, increasing the number of temperature zones has a small effect on system weight, as only additional valves have to be installed. Also, no heat-resistant trim-air 
system is needed, making the architecture more compatible with future more electric aircraft concepts, that work without engine bleed air [2].

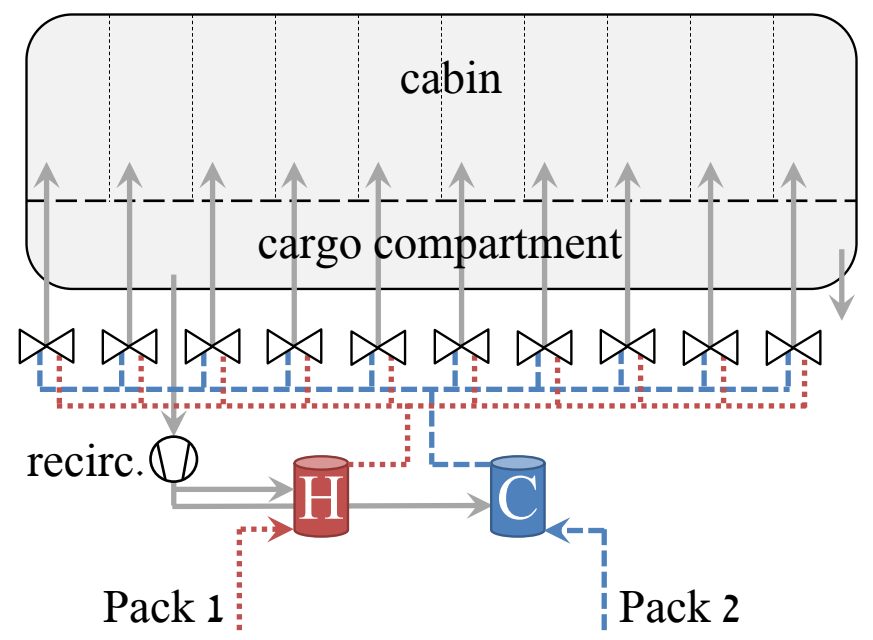

Fig. 3. Alternative cabin temperature regulation architecture (10 temperature zones)

Of course, there is no free lunch. With the conventional architecture, temperature of the individual zones can be controlled independently by adding variable amounts of trim air. With the alternative architecture, strong interaction effects occur: The inlet mass flows in both main pipes are constant, if one of the valves extracts a large amount of air from the cold main pipe. Less cold air is available for the other valves. Also, for an increasing amount of temperature zones the thermal diffusion between neighbouring cabin volumes is much more pronounced.

There are additional reasons that make this system interesting from a control perspective: A small temperature difference between both mixing chambers reduces energy consumption on pack operation [1]. A larger temperature difference gives more control authority to the individual mixing units. It would therefore reduce energy consumption to keep the temperature difference as small as possible (to save energy) and as large as necessary (to be able to reach all target temperatures). Saving energy is the optimization goal, being able to reach all target temperatures is the constraint. On the other hand, this introduces a nonlinearity that the controller has to deal with. Speaking of nonlinearity, in the aviation industry, control systems are kept as simple as possible, to keep certification efforts low [3], [4].

The goal of this paper is to demonstrate a control strategy for the proposed architecture that satisfies the requirements of performance, energy conservation, and simplicity. This paper is structured as follows: In Section II, the control problem is discussed in detail. Section III presents several strategies to control the proposed aircraft-cabin temperature-system. The corresponding results are shown in Section IV. In Section $\mathrm{V}$, the proposed strategies and their results are discussed. Section VI concludes the paper.

\section{PROBLEM DEFINITION}

In this work, an aircraft cabin comprising 10 temperature zones is investigated. For each zone, the air temperature is measured. Setpoint is 20 degrees celcius for each zone. Control inputs are the 10 mixing valves as well as temperature demands for the air conditioning packs. The valves are assumed to be fast. The packs are assumed to have a first-order behavior with a time constant of 10 seconds, additionally their slew rate is limited to 1 Kelvin per second.

The following challenges for the control system were identified:

\section{A. Competition}

Air mass flow through both main pipes is constant. The valves can only redistribute the total mass flow between the temperature zones. If a zone recieves an above average amount of cold air, the others recieve less. If all valves open up, the net result is zero.

It is therefore important, that the valves don't work against each other. If the average temperature in the main pipes is too high, the average cabin temperature will get too high as well, irrespective of the behavior of the valves. Decentralized control strategies would result in a situation, where all valves move in parallel. Since the distribution of air is only dependent on the relative valve positions, the net result is zero.

Thermal diffusion between the different temperature zones tends to bring the temperatures of neighboring temperature zones together. This is helpful for the rejection of disturbances that act on one of the zones. It is counterproductive when the temperature setpoint of neighboring zones diverges. In any case, centralized schemes should have an easier time exploiting this effect.

\section{B. Pack Energy}

A related problem is the cabin energy balance. Since the mass flow rates in both main pipes are fixed and equal, the average temperature of the air in both pipes has to be controlled by regulating the pack outlet temperature demands. The resulting dynamics can interact with the control of the valves, as both packs and valves have a direct influence on the temperature of the cabin zones.

Also, to save fuel the temperature difference between both pipes should be minimized [1]. However, a minimum temperature difference is necessary for the temperature regulation concept to work. This minimum temperature difference is dependent on the current state of heat-loads and temperatures inside the cabin. A suitable controller should therefore keep the temperature difference as small as possible and as large as necessary.

\section{Variable Gain}

Movement of one of the valves has a direct first-order effect on the temperature in the respective zone. This effect is proportional to the temperature difference of the air in both main pipes. Therefore the valves have variable gain, introducing a nonlinearity in the plant. 


\section{METHOD}

In the following, two basic approaches are presented. Additionally a nonlinear compensated variant is described for each basic approach.

\section{A. PID}

Controllers of aircraft energy systems are usually kept simple, often resorting to PID-controllers as first introduced 95 years ago by [5], or the even simpler PI-variants. Staying true to this tradition, the first control approach is completely based on PID-controllers, see Figure 4.

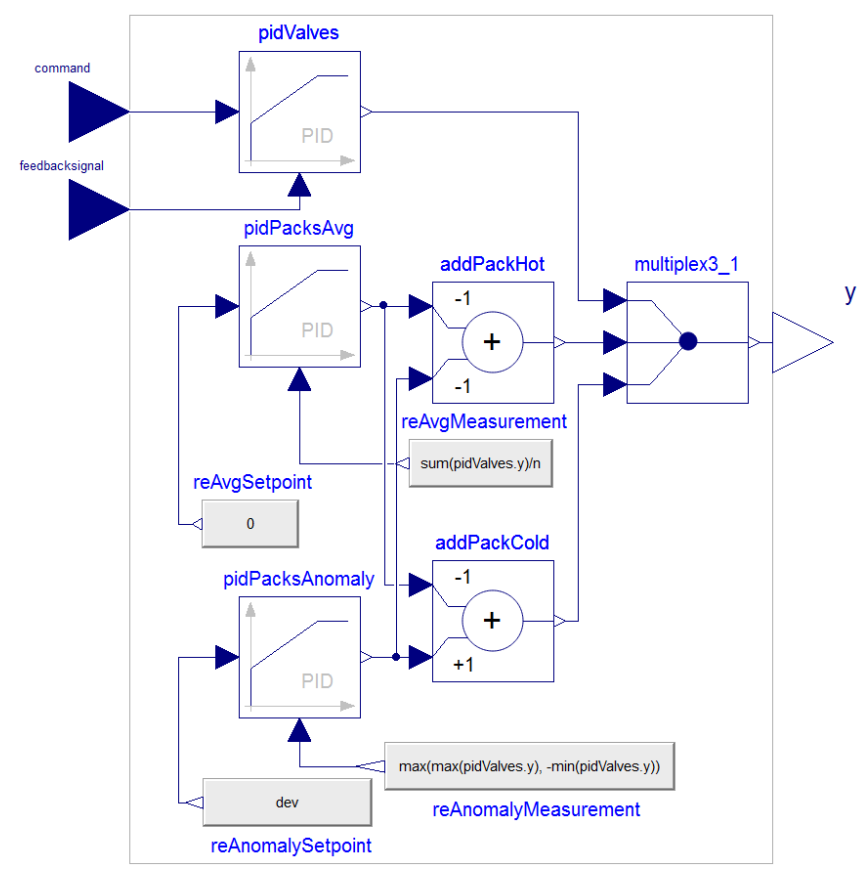

Fig. 4. PID control approach

The approach features a cascading structure. For $n$ cabin zones, $n+2$ PID-controllers are used.

One PID-controller per cabin zone is dedicated to control the respective valve. This group of controllers is called Valvecontrollers. The working range of the valves is mapped to a range of -1 (only cold air) to 1 (only warm air). Accordingly, the controllers are limited to this range, employing an antiwindup track.

Two additional PID-controllers are used to control the pack demand temperatures. The first one of them controls the average pack temperature demand and is therefore called Pack-average-controller. It tries to bring the average output of the Valve-controllers to zero. If the average output of the Valve-controllers is above zero, it means that the average temperature of the complete cabin is too low. The average pack temperature then has to be increased.

The last controller regulates the difference between both pack temperature demands and is therefore called Packanomaly-controller. It is tasked with keeping the pack temperature anomaly as small as possible and as large as necessary. This is realized by the following strategy: For all Valve-controllers, the outputs are continously monitored, and the output with the largest absolute value is computed. If this output is larger then a fixed set-point (for instance 0.8 ), the temperature anomaly is increased. This gives more control authority/gain to the valves, so the output of the Valve-controllers should drop.

The outputs of the Pack-average-controller and the Packanomaly-controller are then combined to find the temperature demands for both air conditioning packs.

\section{B. PID with Control-Input-Normalization}

The PID-approach as described in Subsection III-A is a linear controller for a nonlinear system. However, it is easily possible to compensate for one important nonlinearity: The influence of the control valve positions on the cabin temperature is dependent on the temperature difference of the main pipes. The output of the Valve-controllers can therefore be multiplied with the inverse of the temperature difference of the mixing chambers, thereby compensating for this effect. A correspondingly modified version of the limited PID-controller of the Modelica Standard Library is shown in Figure 5.

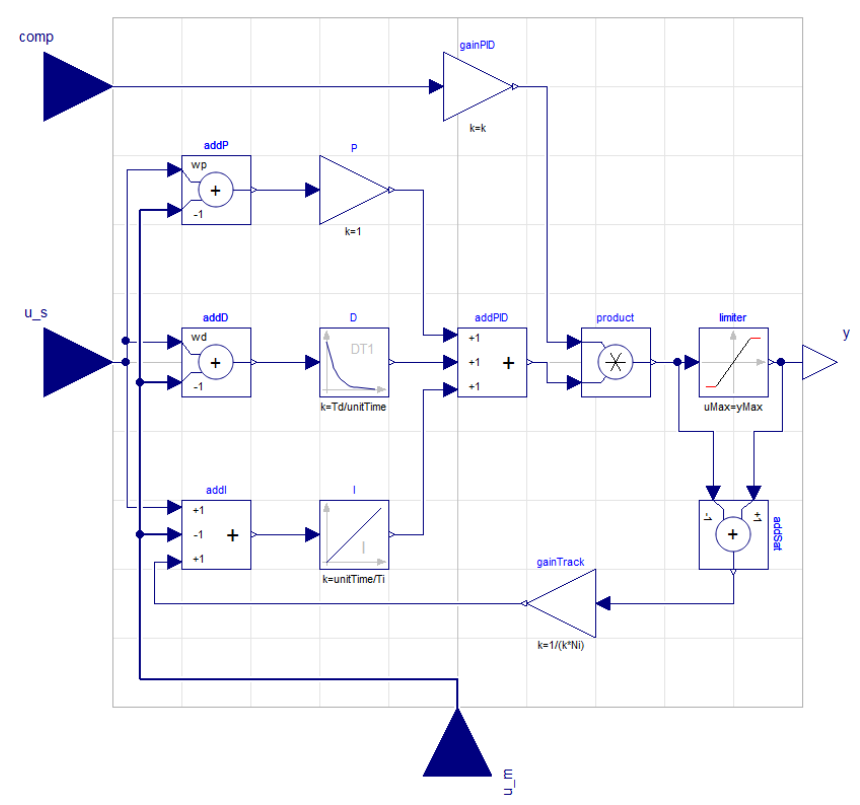

Fig. 5. PID with Control-Input-Normalization control approach

It is important to normalize the PID-controller-output at the correct location. The normalization should take place after the integral element because it affects the actuation - not the measurement. Controller output still has to conform to actuator limits after normalization, so anti-windup measures should be located behind the normalization.

\section{C. $L Q G$}

As already discussed, there are strong interaction effects between the valves. To take advantage of this, an obvious way is to use a centralized control scheme. In this work, an LQG-controller was used. Controller synthesis included 
artificial integrators to assure zero steady-state error, using the formulation as described in [6, p. 348]. Since LQG is based on simulation models, the model as described in [1] was linearized and used.

To keep the number of tuners feasible, most LQG weighting matrices were defined as scalar matrizes. The input cost matrix $\mathrm{R}$ was defined as a block diagonal matrix of two scalar matrices. In this way, weights for valve actuators and pack actuators can be tuned separately. The state cost matrix $\mathrm{Q}$ was defined as a combination of projected system outputs, system states, and artificial integrator states:

$$
\begin{aligned}
& Q=\left(\begin{array}{cc}
w_{\text {states }} I+w_{\text {out }} C^{T} I C & 0 \\
0 & w_{\text {int }} I
\end{array}\right) \\
& R=\left(\begin{array}{cc}
w_{\text {valves }} I & 0 \\
0 & w_{\text {packs }} I
\end{array}\right)
\end{aligned}
$$

The resulting controller takes the form of a single statespace system.

\section{Augmented $L Q G$}

For this approach, several aspects of the other approaches are combined. The first part of the controller is an LQG controller as defined in Subsection III-C. The outputs of the LQG are divided into two groups: Valve positions and Pack demand temperatures. The valve positions are multiplied with a normalization factor which is dependent on the mixing chamber temperatures, similar to the approach described in Subsection III-B. The pack demand temperatures from the LQG controller are averaged, replacing the Pack-averagecontroller as described in Subsection III-A. A PID-controller is added to control the Pack anomaly, similar to the one described in Subsection III-A. This is illustrated in Figure 6. LQE denotes the Linear Quadratic Estimator (Kalman Filter), while LQR denotes the Linear Quadratic Regulator. The combination of both elements is called Linear Quadratic Gaussian Control (LQG).

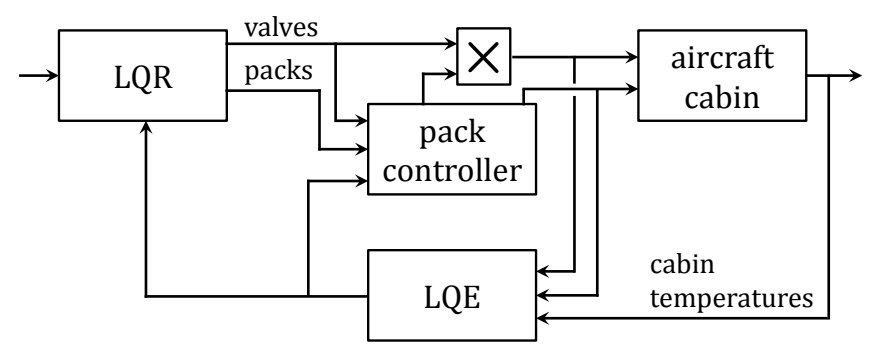

Fig. 6. Augmented LQG control approach

\section{RESULTS}

A simulation model of the aircraft-cabin temperature system was developed in the object-oriented equation-based modelling language Modelica, for details see [1].

As an optimization criterion, a weighted sum of the integrated quadratic control errors, integrated absolute valve movement and pack energy usage was used. This criterion was computed for the case of a standard flight profile including some disturbances in heat-loads.

The PID-based control approaches were implemented in Modelica. The respective tuners were optimized using the Modelica Optimization library [7].

For the synthesis of the LQG-based control approaches, the model was linearized using the Modelica linear systems library as presented in [8]. Also, the nonlinear model was exported, so the optimization criterion could be computed in Simulink. Optimization was done using MOPS [9].

Both optimization tools were configured to use the NelderMead Algorithm [10].

\section{A. Performance}

Selected simulation results for the four optimized control approaches can be seen in Figure 7. The corresponding performance metrics can be seen in Table I.

The PID with Control-Input-Normalization Controller approach offers the best nominal performance followed by the basic PID controller, the Augmented LQG controller and the basic LQG controller.

\section{B. Robustness}

Preliminary simulations show, that the main reason for potential instabilities of the controlled plant is the limited slope of the air conditioning packs. Conventional stability margin analysis is therefore not applicable for the nonlinear system in a meaningful way. Instead, three cases were selected to demonstrate controller performance under parameter changes.

1) Low time constants: In this case, all physical time constants such as air volumes, heat capacities and the timeconstants of the air conditioning pack dynamics are divided by two. This can reflect modelling errors, where the system behaves faster then assumed, as well as situations, where fewer seats or other pieces of furniture are installed by the airline.

2) High time constants: Similar to the previous case but all time constants are multiplied by two instead.

3) Faulty valves: The mass flow of the valves in zones 5 and 7 is set to zero. This reflects a failure situation where the corresponding valves are clogged or failing.

\section{Summary}

The values of the optimization criterion for all control approaches and scenarios are shown in Table I. Selected simulation outputs for the nominal case are shown in Figure 7. Selected simulation outputs for the case faulty valve case are shown in Figure 8.

\section{DISCUSSION}

All of the investigated approaches easily find a stabilizing controller. It is apparent that the adoption of Control Input Normalization has a significant positive effect on controller performance. For each combination of basic control approach and simulation case, the variants using Control Input Normalization offer better performance.

In the nominal case, the PID-based approaches offer superior performance compared to the LQG-based approaches. 
heat sources during flight for different cabin zones
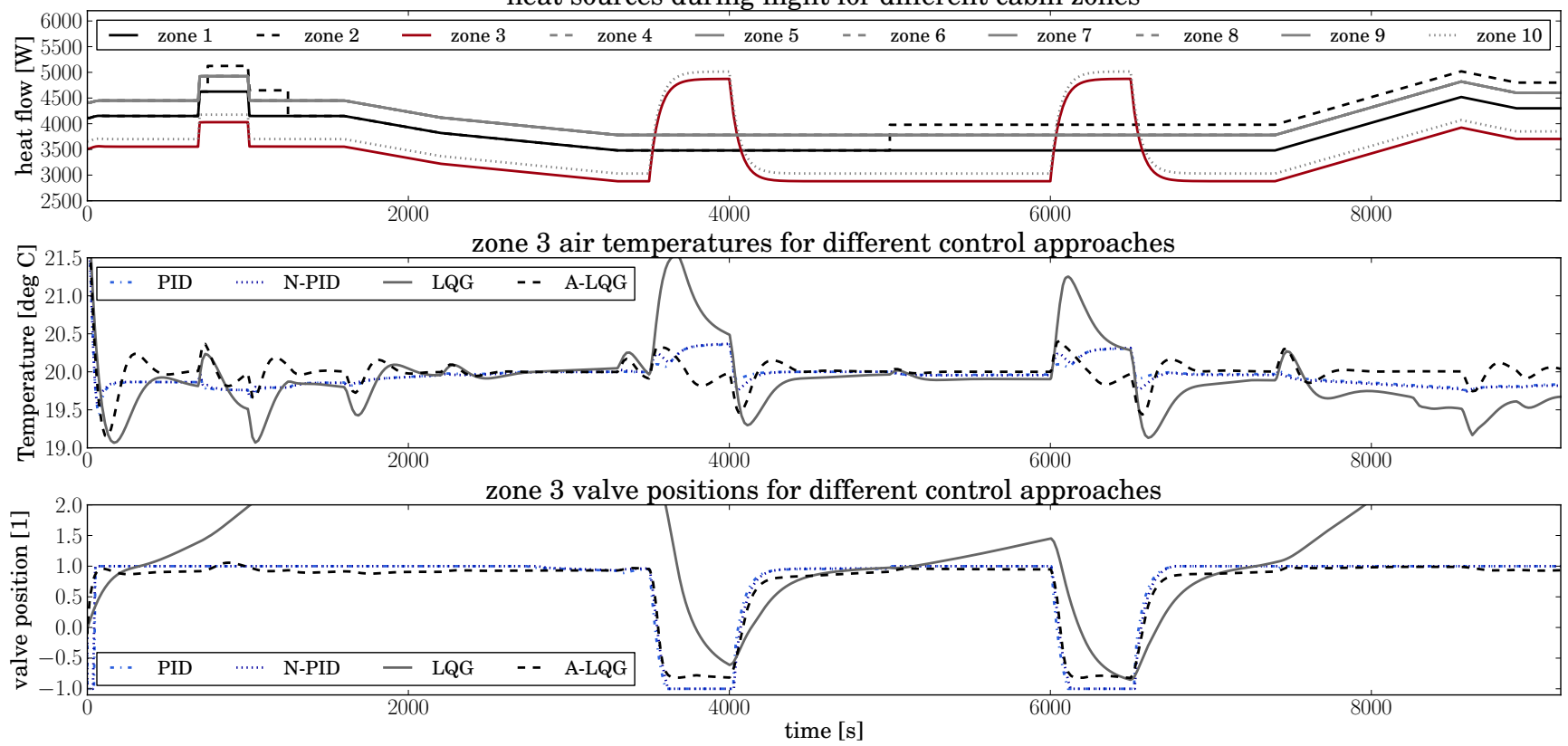

Fig. 7. Simulation results for optimized control approaches - standard case

\begin{tabular}{|c|c|c|c|c|c|c|c|c|c|}
\hline & Competition & Energy & Variable Gain & Anti-Windup & Simplicity & $\begin{array}{l}\text { Number } \\
\text { of tuners }\end{array}$ & $\begin{array}{l}\text { Controller } \\
\text { time states }\end{array}$ & $\begin{array}{l}\text { Performance } \\
\text { (less is better) }\end{array}$ & $\begin{array}{l}\text { Robustness } \\
\text { (less is better) }\end{array}$ \\
\hline PID & - & + & - & + & + & 10 & 24 & 1.079 & $\begin{array}{llll}1.039 & 1.035 & 1.261\end{array}$ \\
\hline N-PID & - & + & + & + & o & 11 & 24 & 1.000 & $1.000 \quad 1.000 \quad 1.255$ \\
\hline LQG & + & - & - & - & o & 7 & 38 & 1.663 & 1.5491 .2291 .190 \\
\hline A-LQG & + & + & + & - & - & 12 & 40 & 1.143 & 1.0361 .0111 .000 \\
\hline
\end{tabular}

TABLE I

SUMMARY OF CONTROL APPROACHES, THEIR FEATURES AND SIMULATED PERFORMANCE, AS DEFINED BY THE METRIC DESCRIBED IN SECTION IV

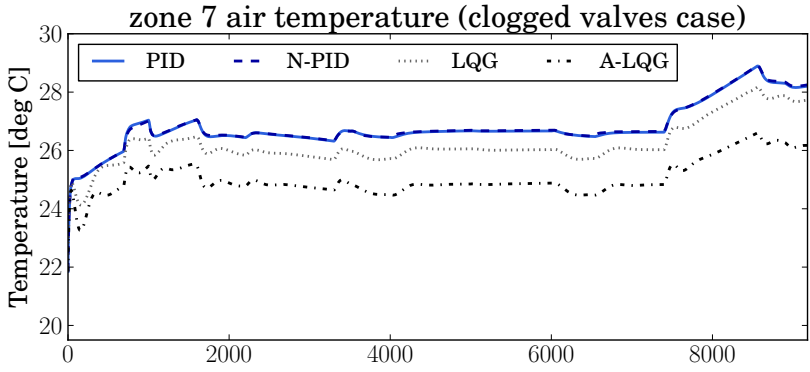

Fig. 8. Simulation results for robustness test with clogged valves in zones 5 and 7

This is unexpected since they have less degrees of freedom. The discrepancy may be caused by the anti-windupcompensation included in the PID-controllers, as well as incomplete tuning of the LQG-based controllers.

For the cases with lower or higher physical time constants the distance in performance between PID-based and LQGbased approaches is significantly smaller. The normalized versions of each approach only differ by 3.6 and 1.1 percent respectively. This relative improvement of the LQG-based approaches may be explained by the large robustness margins of linear quadratic regulators, with LQ regulators having at least 60 degrees phase margin and at least 50 percent gain reduction tolerance [11]. While these guarantees do not hold in the more general LQG case (see the very short abstract of [12]), the influence of the estimator on the robustness seems to be negligible in this case.

In the case of valve failures, LQG-approaches show significantly better behavior. This shows the advantages of centralized control, which exploits interaction effects between neighboring temperature zones.

In this work, the response characteristic of air conditioning packs was realistically modelled, while the control valves were assumed to be infinitely fast. Of course, this does not hold in reality. The different control approaches exhibit varying requirements on the control valve velocities. The maximum valve velocities occuring for the nominal case are shown in Table V.

It is apparent, that the maximum demanded velocity is about an order of magnitude slower for the LQG-based approaches. This allows the use of slower and cheaper valves as well as lighter valve actuators. 


\begin{tabular}{lllll}
\hline Control approach & PID & N-PID & LQG & A-LQG \\
\hline Maximum valve velocity & $18.3 \% / \mathrm{s}$ & $6.9 \% / \mathrm{s}$ & $0.8 \% / \mathrm{s}$ & $1.7 \% / \mathrm{s}$ \\
\hline
\end{tabular}

TABLE II

MAXIMUM OCCURING VALVE VELOCITIES FOR NOMINAL CASE

To summarize, the Augmented LQG approach offers a little worse performance compared with the Normalized PID approach in the nominal case, but equal or better performance during off-design cases. Also, its employment allows for the use of cheaper and lighter valves.

\section{CONCLUSIONS}

For a novel aircraft-cabin temperature-system several control approaches were developed and compared. It became apparent that Control-Input Normalization for the valve actuators resulted in significantly better performance. However, the choice of the control approach is somewhat ambiguous. PIDbased approaches show better nominal performance, LQGbased approaches show better robustness.

\section{ACKNOWLEDGMENT}

Trey and Matt are to thank for their inspiration.

\section{REFERENCES}

[1] A. Pollok, D. Bender, I. Kerling, and D. Zimmer, "Rapid development of an aircraft cabin temperature regulation concept," in 12th International Modelica Conference, 2017.

[2] J. Rosero, J. Ortega, E. Aldabas, and L. Romeral, "Moving towards a more electric aircraft," IEEE Aerospace and Electronic Systems Magazine, vol. 22, no. 3, pp. 3-9, 2007.

[3] S. Jacklin, "Closing the certification gaps in adaptive flight control software," in AIAA Guidance, Navigation and Control Conference and Exhibit, p. 6988, 2008.

[4] M. Steinberg, "Historical overview of research in reconfigurable flight control," Proceedings of the Institution of Mechanical Engineers, Part G: Journal of Aerospace Engineering, vol. 219, no. 4, pp. 263-275, 2005.

[5] N. Minorsky, "Directional stability of automatically steered bodies," Naval Engineers Journal, vol. 32, no. 2, 1922.

[6] S. Skogestad and I. Postlethwaite, Multivariable feedback control: analysis and design, vol. 2. Wiley New York, 2007.

[7] A. Pfeiffer, "Optimization library for interactive multi-criteria optimization tasks," in Proceedings of the 9th International MODELICA Conference; September 3-5; 2012; Munich; Germany, no. 76, pp. 669680, Linköping University Electronic Press; Linköpings universitet, 2012.

[8] M. Baur, M. Otter, and B. Thiele, "Modelica libraries for linear control systems," in Proceedings of 7th International Modelica Conference, Como, Italy, September, pp. 20-22, 2009.

[9] H.-D. Joos, "Multi-objective parameter synthesis (mops)," in Robust Flight Control, pp. 199-217, Springer, 1997.

[10] J. A. Nelder and R. Mead, "A simplex method for function minimization," The computer journal, vol. 7, no. 4, pp. 308-313, 1965.

[11] M. Safonov and M. Athans, "Gain and phase margin for multiloop lqg regulators," IEEE Transactions on Automatic Control, vol. 22, no. 2, pp. 173-179, 1977.

[12] J. Doyle, "Guaranteed margins for lqg regulators," IEEE Transactions on Automatic Control, vol. 23, no. 4, pp. 756-757, 1978. 